\title{
Cervicogenic Dizziness in an II-Year-Old Girl: A Case Report
}

\author{
Eric Chun-Pu Chu $\mathbb{D}^{\prime}$ \\ Arnold Yu-Lok Wong $\mathbb{D}^{2}$ \\ 'New York Chiropractic and \\ Physiotherapy Centre, Hong Kong SAR, \\ People's Republic of China; ${ }^{2}$ Department \\ of Rehabilitation Sciences, The \\ Hong Kong Polytechnic University, \\ Hong Kong SAR, People's Republic of \\ China
}

\begin{abstract}
Cervicogenic dizziness (CGD) is a syndrome of neck pain accompanied by a false sensation of unsteadiness and dizziness due to neck pathology. An 11-year-old girl presented with neck pain and dizziness for four months. According to the patient's statement, her complaints were likely related to the prolonged smartphone use for texting. Sagittal radiograph showed cervical kyphosis, anterior wedging of several vertebrae, and mild anterolisthesis of $\mathrm{C} 2$ on $\mathrm{C} 3$ and $\mathrm{C} 3$ on $\mathrm{C} 4$. These findings might be present in as physiological variants in children. However, continuous static stress in the minor variants could aggravate biomechanical problems, such as cervicogenic dizziness. After ruling out other neurological or vestibular problems, a multicomponent approach consisted of thermal ultrasound therapy, cervical manipulation, and intermittent motorized cervicothoracic traction to release cervical complaints. Three months later, the patient reported a resolution of neck pain and dizziness. At 12-month follow-up, all radiographic metrics showed improvement, including restoration of cervical alignment and lordotic curvature. The immature growing cervical spine has unique anatomic, physiologic and biomechanical features. A static neck flexion can lead to typical injury patterns seen in this age group. This article aims to raise awareness of the potential harms of excessive smartphone use by children.
\end{abstract}

Keywords: anterolisthesis, cervical kyphosis, cervicogenic dizziness, children, excessive smartphone use, neck pain

\section{Introduction}

Cervicogenic dizziness (CGD) is a syndrome of neck pain accompanied by a false sensation of unsteadiness and dizziness due to neck pathology. ${ }^{1,2}$ Despite patients' distressing circumstances, CGD as an independent entity remains controversial. ${ }^{3,4}$ There is no consensus regarding its pathophysiology. Many reported cases of CGD have been associated with whiplash injuries, neck pathologies, poor neck posture, or dysfunctions of the cervical spine. ${ }^{1}$ The neck proprioceptive input, mostly originating from the cervical muscle spindles and joint mechanoreceptors, attests to the head position relative to the trunk. An integration of neck proprioceptive, vestibular and visual sensory inputs contributes to position changes and balance controls in physiological conditions. ${ }^{5,6}$ Disrupted (mechanical dysfunctions) and aberrant (pain) proprioceptive signals from the neck to the vestibular nucleus can result in an inaccurate depiction of head and neck orientation in space, which leads to dizziness. ${ }^{1,7,8}$ CGD can be difficult to diagnose because there are no specific tests to confirm it. However, there are diagnostic criteria that may help to identify CGD: ${ }^{1,2}$ 1) the presence of neck pain before or at the same time of feeling
Correspondence: Eric Chun-Pu Chu New York Chiropractic and Physiotherapy Centre, 4I/F Langham Place Office Tower, 8 Argyle Street, Hong Kong SAR, People's Republic of China

Tel +852-3594-7844

Fax +852-3594-6193

Email eric@nymg.com.hk 
dizzy; 2) successful management of cervical dysfunction leads to a simultaneous relief of dizziness. ${ }^{1,9}$

The immature growing cervical spine has unique anatomic, physiologic and biomechanical features, such as higher ligamentous laxity, malleable features of the vertebra and more horizontal orientation of the facet complex. ${ }^{10}$ Prolonged, excessive neck flexion may predispose children to physical health problems, such as musculoskeletal neck pain, ${ }^{11}$ cervical disc degeneration, ${ }^{12}$ and cervical kyphosis. ${ }^{13}$ The current study serves as a vivid example of identifying CGD and aims to raise awareness of the potential harms of excessive smartphone use by children.

\section{Case Report}

An 11-year-old girl sought chiropractic care because of intermittent dizziness accompanied by neck pain and occipital headache for 4 months. The patient noticed that her headache and neck pain started only while texting on a smartphone. It even seemed that when her neck hurt, she felt dizzy as well. Each episode usually lasted one to two hours. She denied any history of trauma, hearing or vision complaints. Prior neuro-otological examinations (past pointing, nystagmus assessments and caloric testing) and cochlear function tests (otoacoustic emissions testing) excluded vestibular problems or otologic disorders. She had been taking antihistamines and ibuprofen over the past three months. She also underwent rehabilitation exercise and acupuncture for relief from mechanical neck pain, but they were not helpful.

Upon initial evaluations, the patient presented with a guarded neck posture and straight neck. There were no physical signs of stress such as sleeping problems, loss of appetite and difficulty concentrating. The self-reported peak pain intensity of her neck pain and headache was 6 out of 10 on an 11-point numeric pain rating scale. ${ }^{14}$ The Dizziness Handicap Inventory (DHI) questionnaire was scored at 36/100 (mild, 0-30; moderate, 31-60; and severe, $61-100) .{ }^{15}$ There was protective muscle spasm around the neck. Her cervical range of motion was painful at $20^{\circ}$ passive extension (normal: $>60^{\circ}$ ) and restricted at $60^{\circ}$ of bilateral passive rotations (normal: $>80^{\circ}$ ). Tenderness was identified in bilateral upper trapezius, sternocleidomastoid, right rhomboid, and right levator scapulae muscles. Segmental joint motion as assessed by palpation showed restricted at $\mathrm{C} 1 / 2, \mathrm{C} 5 / 6$, $\mathrm{C} 7 / \mathrm{T} 1$, and T1/2 levels. Neurologic examination showed no abnormalities.
Sagittal radiograph (Figure 1A) demonstrated a reversed cervical lordosis. Global cervical lordosis, ${ }^{16}$ defined as Cobb's angle between $\mathrm{C} 2$ and $\mathrm{C} 7$ inferior endplate, was $22^{\circ}$. Several of the vertebrae were wedge-shaped, allowing for slippage (pseudosubluxation or physiological anterolisthesis) $^{17}$ of the $\mathrm{C} 2$ on $\mathrm{C} 3$ and $\mathrm{C} 3$ on $\mathrm{C} 4$, which could be seen as normal variants in children. Intraspinous distance (dashed blue lines) was within 1.5 times the distance of the level either above or below, and the spinous processes of $\mathrm{C} 1$ to $\mathrm{C} 3$ (dashed red line) was aligned within $1 \mathrm{~mm}$ of each other, suggestive of an integrity of the interspinous ligaments. ${ }^{18}$ Diagnosis of cervicogenic dizziness was derived from the patient's history, clinical syndrome of neck pain accompanied by dizziness, an inversion of physiological cervical lordosis, and excluding all other potential causes of dizziness.

Chiropractic intervention consisted of spinal manipulation of the restricted cervical segments, thermal ultrasound therapy to release neck stiffness and restore motion, along with cervicothoracic intermittent motorized traction to release intervertebral spaces and decompress neural impaction. Treatment sessions were arranged three times a week for three months. The patient reported that her pain and dizziness gradually ameliorated from the second week onward and resolved completely within four weeks. Neck mobility was mostly regained near the end of treatment. As a result, the patient reported neck pain reduced from 6/ 10 to $0 / 10$ on pain rating scale. The DHI score declined from 36 to 0 . She continued to receive maintenance treatment focusing on the correction of cervical misalignment on a weekly basis for the ensuing 9 months. Improved cervical alignment and curvature was observed on the 12month follow-up radiograph. With reference to the initial image (Figure $1 \mathrm{~B})$, there was $31^{\circ}\left(-22^{\circ}\right.$ vs $\left.9^{\circ}\right)$ correction of the cervical lordosis. Reduced width of the prevertebral soft-tissue thickening (orange lines) could signify a relief of previously swollen soft-tissue. ${ }^{19}$ The patient was subsequently reviewed at the 18th month and remained symptoms free. No adverse events had occurred during the 12 months of care (Figure 2).

\section{Discussion}

With an erect bipedal posture and a mobile head in human beings, vestibular signals alone cannot distinguish dynamics of the head or of the whole body when the head moves on a stationary trunk. ${ }^{6,20}$ Neck muscles have abundant muscle spindles, particularly in small suboccipital muscles (ie, the semispinalis capitis and cervicis 


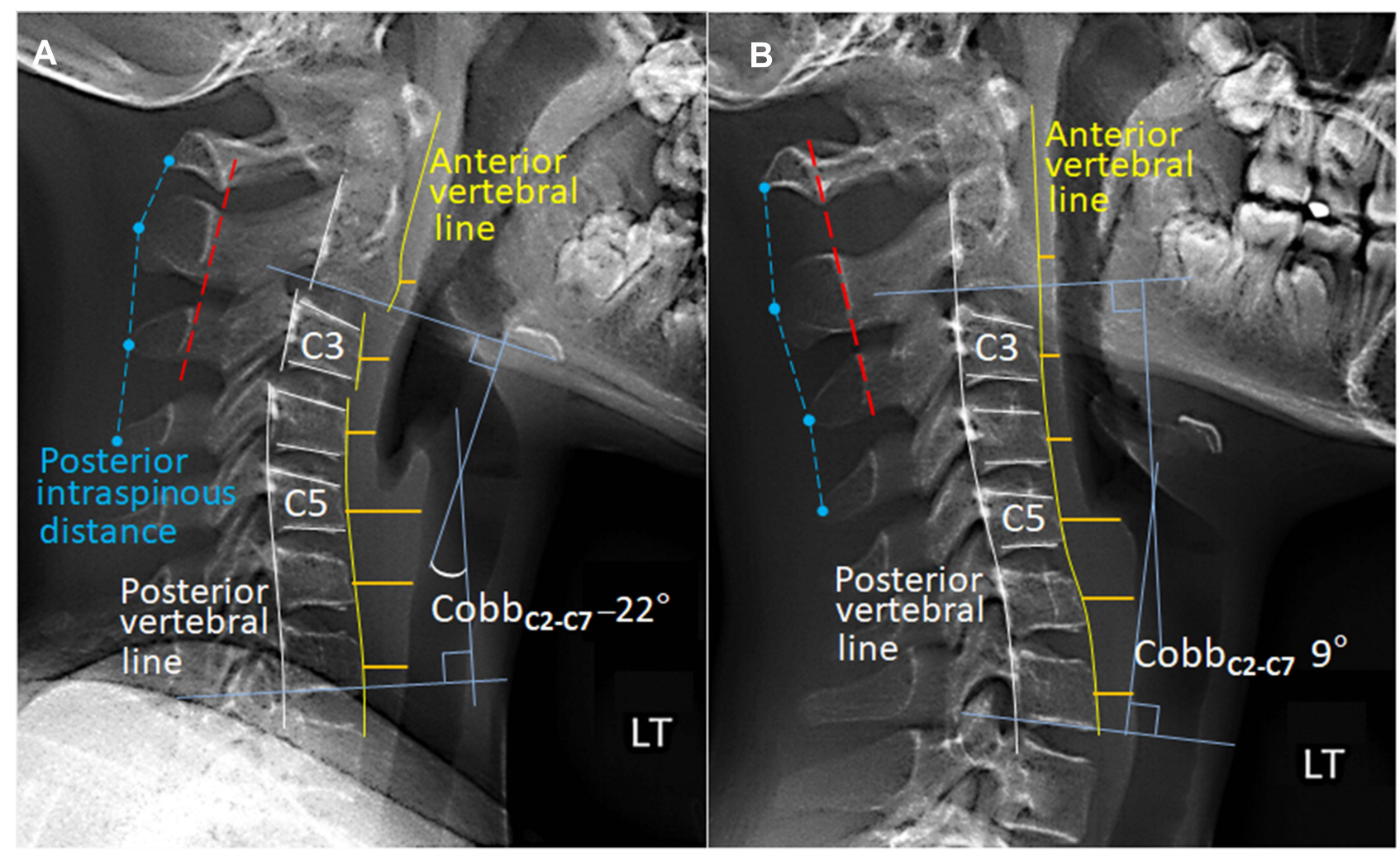

Figure I Comparison of cervical alignment on sagittal radiographs in neutral position. (A) At the initial assessment, several of the vertebrae were wedge-shaped, allowing for slippage of the C2 on C3 and C3 on C4. The global curvature of the C2-C7 spine using Cobb angle (four blue lines) was $-22^{\circ}$, a reversed cervical lordosis. Intraspinous distance (dashed blue lines) was within 1.5 times the distance of the level either above or below and the spinous processes of $\mathrm{Cl}$ to $\mathrm{C} 3$ (dashed red line) was aligned within I mm of each other, suggestive an integrity of the interspinous ligaments. (B) 12-months later, the vertebral configuration became roughly rectangular. Restored cervical alignment and cervical lordosis yielded simultaneous remission of neurological symptoms. With reference to the initial image, there was $31^{\circ}\left(-22^{\circ}\right.$ vs $\left.9^{\circ}\right)$ correction of the cervical lordosis. Reduced width of the prevertebral soft-tissue thickening (Orange lines) could signify a relief of previously swollen soft-tissue.

muscles). ${ }^{8}$ Muscle spindles are stretch receptors in skeletal muscles that sense muscle length and the velocity of muscle length change, contributing to fine motor control and providing axial and limb position information to the central nervous system. Facet capsules are also richly innervated with nociceptors, which estimate the orientation of the head. $^{21,22}$ Cervical diseases can lead to a conflict between muscle perception and joint position sense and a distorted interaction between vestibular and cervical inputs, resulting in a false sensation of unsteadiness. $^{8,21}$

As there is an increasing use of smartphones, the immature growing cervical spine is more prone to kyphotic deformity due to children having a relatively large head, higher ligamentous laxity, immature paraspinal muscles, shallow facet joints, high water content in the disks, unfused epiphyses, and wedging of vertebrae. ${ }^{10,23}$ The fulcrum for flexion in the cervical spine in children is at the $\mathrm{C} 2 / \mathrm{C} 3$ level and descends to $\mathrm{C} 5 / \mathrm{C} 6$ as they grow. ${ }^{18}$ In this context, it is possible to misinterpret these physiologic variants, such as loss of cervical lordosis, wedging or anterior displacement of the vertebrae, as a pathological ramification. ${ }^{18}$ Minor anatomical anomalies that cause no disability or have no significant physical or functional effects can be regarded as physiologic/congenital variants. ${ }^{24}$ However, continuous static stress in the minor variants can aggravate biomechanical alterations and predispose segmental instability of the cervical spine, resulting in CGD.

A static and flexed neck posture due to excessive texting on a smartphone can make the neck muscles go into painful spasm. Neck perception and joint position sense are primarily impaired by compromised muscle perception, joint mechanics, or capsule sensitivity and, secondarily, by disruption of the afferent proprioceptive tuning to the vestibular nucleus. ${ }^{21,25}$ A defective mechanoreceptor's feedback or compromised sensory integration may contribute to a sensation of unsteadiness. ${ }^{3,21}$ Despite the aforementioned hypothesis, a sensation of dizziness as a consequence of cervical spine dysfunctions has not been widely accepted by the medical profession..$^{1,3,25}$ The 


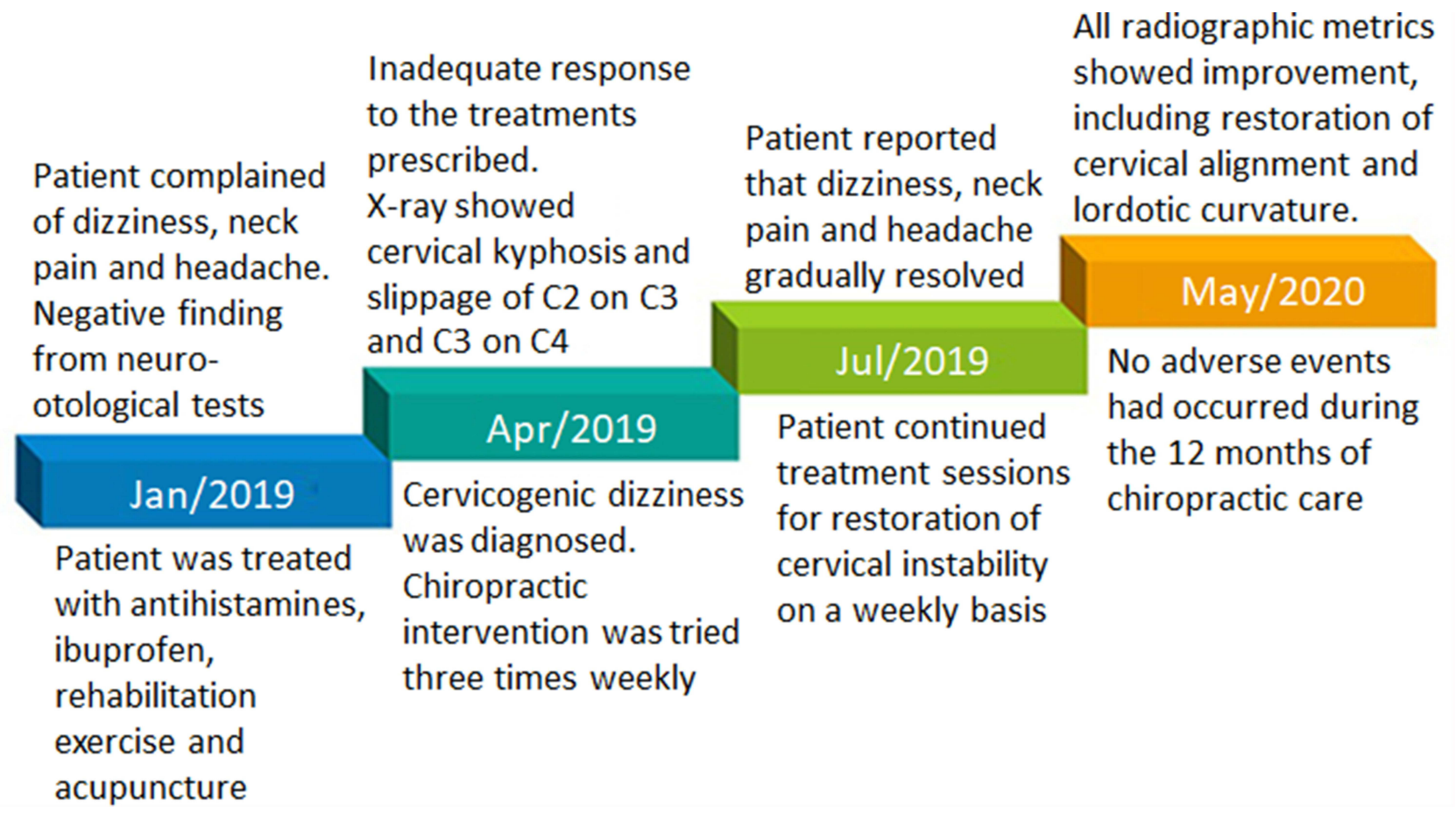

Figure 2 Clinical timeline of the presenting case.

inclusion of CGD as an independent entity still remains controversial. ${ }^{4}$ The overall prevalence of CGD is not known because there are no generally accepted clinical or paraclinical tests for CGD. ${ }^{26}$

As the name might suggest, CGD is a false feeling of dizziness due to cervical musculoskeletal dysfunction. Migraine-related syndromes are the most common cause of episodic dizziness in children. CGD can be differentiated from Vestibular Migraine of Childhood and Recurrent Vertigo of Childhood by the absence of nystagmus and hearing loss. ${ }^{27}$ The correlation between dizzy syndrome and neck movement is an explicit diagnostic criterion for CGD. ${ }^{1,2}$ Symptoms caused by CGD should be aggravated by movements that elicit neck pain and should subside with interventions that alleviate neck pain. ${ }^{1}$ Treatments for CGD should target neck problems and be tailored for individual patients based on their treatment responses. Recent systematic reviews $^{22,28}$ indicate that manual therapy may benefit cervicogenic dizziness but good quality randomized controlled trials are warranted. That said it is noteworthy that manual therapy should be applied with great caution in patients with CGD. Notably, it is critically important to rule out neurovascular aetiologies before starting the manual therapy to prevent any untoward events in CGD. ${ }^{6}$ Surgery is usually not indicated for mechanical causes of neck pain ${ }^{29}$ unless there are progressive neurologic deficits or intractable pain and disability unresponsive to conservative treatments.

\section{Conclusion}

The immature growing cervical spine has unique anatomic, physiologic and biomechanical features, such as higher ligamentous laxity, malleable features of the vertebra and more horizontal orientation of the facet complex. ${ }^{9}$ Excessive smartphone use can result in neck pain and musculoskeletal disorders. Erroneous proprioceptive inputs from various neck problems can result in dizziness. Determining the cause of dizziness is crucial to guide the selection of the most appropriate treatment regimen.

\section{Abbreviations}

CGD, cervicogenic dizziness; DIH, Dizziness Handicap Inventory.

\section{Ethical Approval and Informed Consent}

Institutional Review Board (IRB) approval for this case report was not required. Written informed consent was obtained from the guardian of the patient for publication of this case report and any accompanying images. 


\section{Author Contributions}

All authors made a significant contribution to the work reported, whether that is in the conception, study design, execution, acquisition of data, analysis and interpretation, or in all these areas; took part in drafting, revising or critically reviewing the article; gave final approval of the version to be published; have agreed on the journal to which the article has been submitted; and agree to be accountable for all aspects of the work.

\section{Disclosure}

The authors report no conflicts of interest in this work.

\section{References}

1. Reiley AS, Vickory FM, Funderburg SE, Cesario RA, Clendaniel RA. How to diagnose cervicogenic dizziness. Arch Physiother. 2017;7:12. doi:10.1186/s40945-017-0040-x

2. Chu ECP, Al Zoubi F, Yang J. Cervicogenic dizziness associated with craniocervical instability: a case report. J Med Cases. 2021;12 (11):451-454. doi: $10.14740 / \mathrm{jmc} 3792$

3. Brandt T, Bronstein AM. Cervical vertigo. J Neurol Neurosurg Psychiatry. 2001;71(1):8-12. doi:10.1136/jnnp.71.1.8

4. Li Y, Peng B. Pathogenesis, diagnosis, and treatment of cervical vertigo. Pain Phys. 2015;18(4):E583-E595. PMID: 26218949.

5. Peterka RJ. Sensorimotor integration in human postural control. J Neurophysiol. 2002;88(3):1097-1118. doi:10.1152/jn.2002.88.3

6. Chu ECP, Chin WL, Bhaumik A. Cervicogenic dizziness. Oxf Med Case Rep. 2019;11:476-478. doi:10.1093/omcr/omz115

7. Kang JH, Park RY, Lee SJ, Kim JY, Yoon SR, Jung KI. The effect of the forward head posture on postural balance in long time computer based worker. Ann Rehabil Med. 2012;36(1):98-104. doi:10.5535/ arm.2012.36.1.98

8. Peng B, Yang L, Li Y, Liu T, Liu Y. Cervical proprioception Impairment in neck pain-pathophysiology, clinical evaluation, and management: a narrative review. Pain Ther. 2021;10(1):143-164. doi:10.1007/s40122-020-00230-z

9. Magnusson M, Malmström EM. The conundrum of cervicogenic dizziness. Handb Clin Neurol. 2016;137:365-369. doi:10.1016/ B978-0-444-63437-5.00026-1

10. Chu ECP, Chakkaravarthy DM, Lo FS, Bhaumik A. Atlantoaxial rotatory subluxation in a 10-year old boy. Clin Med Insights Arthritis Musculoskelet Disord. 2020;13:1179544120939069. doi:10.1177/1179544120939069

11. David D, Giannini C, Chiarelli F, Mohn A. Text neck syndrome in children and adolescents. Int J Environ Res Public Health. 2021;18 (4):1565. doi:10.3390/ijerph 18041565

12. Zhuang L, Wang L, Xu D, Wang Z, Liang R. Association between excessive smartphone use and cervical disc degeneration in young patients suffering from chronic neck pain. J Orthop Sci. 2021;26 (1):110-115. doi:10.1016/j.jos.2020.02.009

13. Lai J, Ji G, Zhou Y, et al. Apoptosis of endplate chondrocytes in cervical kyphosis is associated with chronic forward flexed neck: an in vivo rat bipedal walking model. J Orthop Surg Res. 2021;16:5. doi:10.1186/s13018-020-02124-4
14. Jensen MP, McFarland CA. Increasing the reliability and validity of pain intensity measurement in chronic pain patients. Pain. 1993;55:195-203. doi:10.1016/0304-3959(93)90148-I

15. Grigol TA, Silva AM, Ferreira MM, Manso A, Ganança MM, Caovilla HH. Dizziness handicap inventory and visual vertigo analog scale in vestibular dysfunction. Int Arch Otorhinolaryngol. 2016;20 (3):241-243. doi:10.1055/s-0035-1567808

16. Martini ML, Neifert SN, Chapman EK, Mroz TE, Rasouli JJ. Cervical spine alignment in the sagittal axis: a review of the best validated measures in clinical practice. Global Spine J. 2021;11 (8):1307-1312. doi:10.1177/2192568220972076

17. Booth TN. Cervical spine evaluation in pediatric trauma. AJR Am J Roentgenol. 2012;198(5):W417-25. doi:10.2214/AJR.11.8150

18. Lustrin ES, Karakas SP, Ortiz AO, et al. Pediatric cervical spine: normal anatomy, variants, and trauma. RadioGraphics. 2003;23 (3):539-560. doi:10.1148/rg.233025121

19. Vermess D, Rojas CA, Shaheen F, Roy P, Martinez CR. Normal pediatric prevertebral soft-tissue thickness on MDCT. AJR Am J Roentgenol. 2012;199(1):W130-3. doi:10.2214/AJR.10.6288

20. Pettorossi VE, Schieppati M. Neck proprioception shapes body orientation and perception of motion. Front Hum Neurosci. 2014;8:895. doi:10.3389/fnhum.2014.00895

21. Reddy RS, Tedla JS, Dixit S, Abohashrh M. Cervical proprioception and its relationship with neck pain intensity in subjects with cervical spondylosis. BMC Musculoskelet Disord. 2019;20(1):447. doi:10.1186/s12891-019-2846-z

22. Hoppes CW, Romanello AJ, Gaudette KE, et al. Physical therapy interventions for cervicogenic dizziness in a military-aged population: protocol for a systematic review. Syst Rev. 2020;9(1):62. doi:10.1186/s13643-020-01335-4

23. Figaji AA. Anatomical and physiological differences between children and adults relevant to traumatic brain injury and the implications for clinical assessment and care. Front Neurol. 2017;8:685. doi:10.3389/fneur.2017.00685

24. Ameen SK, Alalaf SK, Shabila NP. Pattern of congenital anomalies at birth and their correlations with maternal characteristics in the maternity teaching hospital, Erbil city, Iraq. BMC Pregnancy Childbirth. 2018;18:501.

25. Kristjansson E, Treleaven J. Sensorimotor function and dizziness in neck pain: implications for assessment and management. J Orthop Sports Phys Thery. 2009;39(5):364-377. doi:10.2519/jospt.2009.2834

26. Kadanka Z Jr, Kadanka Z Sr, Jura R, Bednarik J. Vertigo in patients with degenerative cervical myelopathy. J Clin Med. 2021;10 (11):2496. doi: $10.3390 / \mathrm{jcm} 10112496$

27. van de Berg R, Widdershoven J, Bisdorff A, et al. Vestibular migraine of childhood and recurrent vertigo of childhood: diagnostic criteria consensus document of the Committee for the Classification of Vestibular Disorders of the Bárány Society and the International Headache Society. J Vestib Res. 2021;31(1):1-9. doi:10.3233/VES-200003

28. Lystad RP, Bell G, Bonnevie-Svendsen M, Carter C. Manual therapy with and without vestibular rehabilitation for cervicogenic dizziness: a systematic review. Chiropr Man Ther. 2011;19:21. doi:10.1186/ 2045-709X-19-21

29. Evans G. Identifying and treating the causes of neck pain. Med Clin N Am. 2014;98(3):645-661. doi:10.1016/j.mena.2014.01.015 


\section{Publish your work in this journal}

Adolescent Health, Medicine and Therapeutics is an international, peer-reviewed, open access journal focusing on health, pathology, and treatment issues specific to the adolescent age group. All aspects of health maintenance, preventative measures and disease treatment interventions are addressed within the journal and practitioners from all disciplines are invited to submit their work as well as healthcare researchers and patient support groups. The manuscript management system is completely online and includes a very quick and fair peerreview system. Visit http://www.dovepress.com/testimonials.php to read real quotes from published authors. 